Series A

I. MATHEMATICA

$336 / 12$

\title{
THE DISTINGUISHED BOUNDARY SETS AND VALUE DISTRIBUTION OF FUNCTIONS OF TWO COMPLEX VARIABLES
}

BY

STEFAN BERGMAN

HELSIN K I 1963

S U O M A L A I N N TIEDEAKA TEM I A

https://doi.org/10.5186/aasfm.1964.336-12 
Communicated 13 September 1963 by P. J. Myrberg and Oldi Lehto 


\section{The distinguished boundary sets and value distribution of functions of two complex variables*}

\section{The problem of function theoretical characterization of domains in the space of two complex variables}

Every simply connected domain with at least two boundary points can be mapped onto the unit disc. Therefore, the study of value distribution of an analytic function $f(z)$ in a simply connected domain can in most cases be reduced to the investigation of the behavior of the function $f$ in the disc. The situation changes completely if we pass to the case of several complex variables. Two domains of the $z_{1}, z_{2}$-space, $z_{k}=x_{k}+i y_{k}$, $k=1,2$, cannot, in general, be mapped onto each other by a pair of analytic functions $\varphi_{k}\left(z_{1}, z_{2}\right), k=1,2$, of two complex variables; various domains can exhibit completely different behavior of analytic functions $f\left(z_{1}, z_{2}\right)$ defined in these domains. For instance, in the case of the hypersphere $\left.{ }^{1}\right) \mathfrak{H}=\left[\left|z_{1}\right|^{2}+\left|z_{2}\right|^{2}<1\right]$ to every point $\left(z_{1}^{0}, z_{2}^{0}\right)$ of the (three-dimensional) boundary $\mathfrak{h}^{3}=\left[\left|z_{1}\right|^{2}+\left|z_{2}\right|^{2}=1\right]$ a function $f$ exists having the property that

$$
\left|f\left(z_{1}^{0}, z_{2}^{0}\right)\right|>\left|f\left(z_{1}, z_{2}\right)\right|, \quad\left(z_{1}, z_{2}\right) \in \overline{\mathfrak{S}}-\left(z_{1}^{0}, z_{2}^{0}\right),
$$

while in the case of the bicylinder $\mathfrak{C}=\left[\left|z_{1}\right|<1,\left|z_{2}\right|<1\right]$ every $f\left(z_{1}, z_{2}\right)$ holomorphic in $\mathfrak{C}$, continuous in $\overline{\mathfrak{C}}$, assumes the maximum of its absolute value already on the two-dimensional subset $\left[\left|z_{1}\right|=1,\left|z_{2}\right|=1\right]$ of the boundary. It arises the problem to study the "functional theoretical structure» of domains, i. e., to investigate the behavior of analytic functions in different types of domains and to classify them. A method to carry out such a study (followed in the present paper) is to introduce some special domains, namely analytic polyhedra, i. e., domains bounded by finitely many analytic hypersurfaces ${ }^{2}$ ).

* The paper has been done under the grant of the National Science Foundation No. 21344 and Nonr 225(11).

1) German characters are used for manifolds. The superscript "n» indicates the dimension of the manifold for $n=1,2,3$.

$\left.{ }^{2}\right)$ In the following, analytic hypersurfaces will stand for segments of analytic hypersurfaces and analytic surfaces for segments of analytic surfaces. 
(A polyhedron $\mathfrak{B}$ similar to the bicylinder has the property that the analytic function $f\left(z_{1}, z_{2}\right)$, continuous in $\overline{\mathfrak{B}}$, assumes the maximum of its absolute value on the (two-dimensional) distinguished boundary of $\mathfrak{B}$. Concerning various investigations of functions $f$ in polyhedra, see $[2]-[9]$.

An analytic hypersurface $\mathfrak{h}^{3}$ is a one-parameter family of analytic surfaces $\mathfrak{\Im}^{2}(\lambda)$, i. e., $\mathfrak{h}^{3}=\bigcup_{\lambda} \mathfrak{J}^{2}(\lambda), \quad \lambda$ real. We assume that a second analytic surface $\mathfrak{I}^{2}$ intersects $\bigcup_{\lambda} \mathfrak{S}^{2}(\lambda)$ (or a combination of a finite number of $\mathfrak{h}_{*}^{3}$ ) in a closed curve which forms the boundary of the segment $\mathfrak{I}^{2}$. Since in every $\mathfrak{I}^{2}(\lambda)$ and in $\mathfrak{I}^{2}, f\left(z_{1}, z_{2}\right)$ is an analytic function of one complex variable, one can obtain theorems of functions of two complex variables by applying the results in one variable twice. In particular, in this way one derives a generalized Poisson-Jensen formula (see [6][8]) expressing $\log |f(0,0)|$ in terms of the values of $\log |f|$ on the (two-dimensional) surface $\mathfrak{F}^{2}=\bigcup_{\lambda} \mathfrak{i}^{1}(\lambda)$ and in terms of certain functionals connected with the pole and zero surfaces of $f$. Here $\mathfrak{i}^{1}(\lambda)$ is the boundary curve of $\mathfrak{\Im}^{2}(\lambda)$. Suppose that we have a one-parameter family $\mathfrak{F}^{2}(s)=\bigcup_{\lambda} \mathfrak{i}^{1}(s, \lambda), 0<s_{1} \leqq s<\infty$, of surfaces where $\mathfrak{i}^{1}(s, \lambda)$ is the boundary curve of $\mathfrak{\Im}^{2}(s, \lambda)$ (see above). The previous considerations yield relations for the growth of $|f|$ on a three-dimensional manifold $\bigcup_{s=s_{1}}^{\infty} \mathfrak{F}^{2}(s)=\mathfrak{i}^{3}$ and certain functionals connected with the zero and pole surfaces of $f$. In this way, we obtain an analogue of the first Nevanlinna theorem.

Remark. We note that our approach yields relations for the rate of growth of $|f|$ on the three-dimensional manifold $\tilde{\mathfrak{i}}^{3}$ and not in the whole four-dimensional space.

Results of this type are discussed in [7]-[8], and in $\S 3$ of the present paper. In $\S 4$ we show that, using a similar approach, an analogue of Nevanlinna's second theorem can also be derived.

While in $\S \S 2-4$ we consider analytic hypersurfaces $\mathfrak{h}^{3}=\bigcup \mathfrak{\Im}^{2}(\lambda)$, where $\mathfrak{\Im}^{2}\left(\lambda_{1}\right) \cap \mathfrak{\Im}^{2}\left(\lambda_{2}\right)=0$ for $\lambda_{1} \neq \lambda_{2}$, we introduce analytic hypersurfaces in $\S 5$ where all $\mathfrak{\Im}^{2}(\lambda), \quad 0 \leqq \lambda \leqq 1$, have a common intersection point, $O$. In this case we consider in an analytic hypersurface a function $f\left(z_{1}, z_{2}\right)$ which in every lamina $\mathfrak{F}^{2}(\lambda)$ omits two values. Using the Schottky theorem, one obtains bounds for $|f|$ in terms of its value at the point $O$ in a domain bounded by $\mathfrak{h}^{3}$ and another analytic hypersurface.

Finally, in $\S 6$ we discuss the connection between the coefficients of the series development of $f$ and the regularity domain of $f$. In addition 
to the classical methods the use of the theory of orthogonal functions for this purpose is discussed.

The methods used in the present paper can be generalized to the case of $n$ variables, $n>2$. The author will discuss the generalizations in another paper.

\section{A class of domains in the space of two complex variables}

2.1. A segment of an analytic hypersurface. Let $^{3}$ )

(1) $\quad h_{k \varkappa}\left(s ; \hat{Z}_{\varkappa}, t_{\varkappa}, \bar{t}_{\varkappa}\right) \equiv h_{k \varkappa}\left(s ; \hat{Z}_{\varkappa}, t_{\varkappa}\right), \quad \varkappa=1,2, \ldots, m, \quad k=1,2$,

$$
t_{\varkappa}=s_{\varkappa} e^{i \chi_{\varkappa}}, \quad h_{1 \varkappa}\left(s ; 0, t_{\varkappa}\right)=0,
$$

be a family of single-valued, continuously differentiable functions of complex variables $\hat{\mathrm{Z}}_{\varkappa}, t_{\varkappa}, \bar{t}_{\varkappa}$ and continuous in $s, 0<s_{0} \leqq s<\infty$, which are defined in a sufficiently large domain (1a) [hypothesis 1a]. For every fixed $t_{\varkappa}$ the functions $h_{k \varkappa}\left(s ; \hat{\mathbf{Z}}_{\varkappa}, t_{\varkappa}\right)$ are holomorphic in $\left|\hat{\mathbf{Z}}_{\varkappa}\right| \leqq r$ and $h_{2 \varkappa}\left(s ; 0, t_{\varkappa}\right)$ is an entire function of the complex variables $t_{\varkappa}, \bar{t}_{\varkappa}$ (1b). For every fixed $t_{\varkappa}, \quad(1)$ is a one-to-one mapping of $\left|\hat{\mathbf{Z}}_{\varkappa}\right| \leqq r$ onto the analytic surface

(2) $\mathfrak{\Im}_{\varkappa}^{2}\left(t_{\varkappa}\right)=\left[z_{k}=h_{k \varkappa}\left(s ; \hat{Z}_{\varkappa}, t_{\varkappa}, \bar{t}_{\varkappa}\right) \equiv h_{k \varkappa}\left(s ; \hat{Z}_{\varkappa}, t_{\varkappa}\right)\right], \quad \varkappa=1,2, \ldots, m$,

(10). The boundary curve $\mathfrak{i}_{\varkappa}^{1}\left(t_{\varkappa}\right)$ of $\mathfrak{\Im}_{\varkappa}^{2}\left(t_{\varkappa}\right)$ is the image of $\left|\hat{\mathbf{Z}}_{x}\right|=r$ (1d). The positive direction on $i_{\%}^{1}\left(t_{r}\right)$ corresponds to the direction for which $\left|\hat{Z}_{\varkappa}\right|<r$ lies on the left-hand side of $\left|\hat{\mathbf{Z}}_{\varkappa}\right|=r$ if $\arg \hat{\mathbf{Z}}_{\varkappa}$ increases (1e).

Let $t_{\varkappa}=s_{\varkappa} e^{i \varkappa_{\varkappa}}$, where $s_{\varkappa}$ is a fixed value. For a given $\varkappa$ the family

$$
\Im_{\varkappa}^{2}\left(s_{\varkappa} e^{i \chi_{\varkappa}}\right), \quad \chi_{\varkappa 1} \leqq \chi_{*} \leqq \chi_{\varkappa 2}
$$

has the following properties:

$$
\mathfrak{\Im}_{\varkappa}^{2}\left(s_{\varkappa} e^{i \chi_{\varkappa \nu}}\right) \cap \mathfrak{\Im}_{\varkappa}^{2}\left(s_{\varkappa} e^{i \chi_{\varkappa \mu}}\right)=\varnothing, \quad \chi_{\varkappa 1} \leqq \chi_{\varkappa \nu}<\chi_{\varkappa \mu}<\chi_{\varkappa 2} .
$$

$\left.{ }^{3}\right)$ In this section and in the first part of $\S 3$ the quantities $s, s_{\varkappa}$ and $r$ are constants. In the second part of $\S 3$ (see p. 9) and in $\S 4$, we assume that $s_{r}$ and $r$ are monotone functions of $s$ and we investigate the behavior of certain functionals (described in the following) for $s \rightarrow \infty$. We indicate in some cases that various sets and functionals depend on $s, s_{\varkappa}$ and $r$, but to avoid to cumbersome a notation, often we omit to do this. 
A. I. $336 / 12$

There are two possibilities:

$$
\mathfrak{J}^{2}\left(s_{\varkappa} e^{i \chi_{\varkappa 1}}\right) \bigcap \mathfrak{\Im}^{2}\left(s_{\varkappa} e^{i \chi_{\varkappa 2}}\right)=\varnothing
$$

or

$$
\mathfrak{J}^{2}\left(s_{\varkappa} e^{i \chi_{\varkappa \mathrm{i}}}\right)=\mathfrak{S}^{2}\left(s_{\varkappa} e^{i \chi_{\varkappa 2}}\right) .
$$

In case $(5 b)$ the sense of direction of $\lim \dot{i}_{\gamma_{\varkappa}}^{1}\left(s_{\varkappa^{\prime}} e^{i \chi_{\varkappa}}\right)$ is the same as that of $\mathfrak{i}_{\varkappa}^{1}\left(s_{\varkappa} e^{i \chi_{\varkappa}}\right)$.

\section{Definition.}

$$
\mathfrak{e}_{\varkappa}^{3}=\bigcup_{\chi_{\varkappa}=\chi_{\varkappa 1}}^{\chi_{\varkappa 2}} \mathfrak{\Im}_{\varkappa}^{2}\left(s_{\varkappa} e^{i \gamma_{\varkappa}}\right)
$$

is called a segment of an analytic hypersurface. $\mathfrak{I}_{\varkappa}^{2}\left(s_{\varkappa} e^{i \chi_{\varkappa}}\right)$ is called a lamina of $\mathfrak{c}_{\varkappa}^{3}$.

Remark 1. In addition to hypersurfaces satisfying the condition (4) there exist hypersurfaces such that different laminas have intersection points. See $\S 5$ and $\S 6$.

2.2. The boundary of the domain $\mathfrak{B}(s)$. Let $\mathfrak{B}=\mathbf{B}(s)$ denote a bounded domain in the $z_{1}, z_{2}$-plane.

We assume that the boundary of the domain $\mathbf{B}(s)$ consists of finitely many segments $\mathfrak{e}_{x}^{3}, \quad x=1,2, \ldots, m$, and of a segment of a hypersurface $\mathfrak{b}_{0}^{3} . \quad \mathfrak{b}_{0}^{3}$ is a connected manifold which consists of finitely many continuously differentiable (three-dimensional) submanifolds (2a).

Let

$$
\mathfrak{F}_{\varkappa \mu}^{2}=\mathfrak{e}_{\varkappa}^{3} \cap \mathfrak{e}_{\mu}^{3}, \quad x \neq \mu, \quad x=1,2, \ldots, m,
$$

(2b). Further we assume that

$$
\mathfrak{F}_{\varkappa}^{2}=\bigcup_{\substack{\mu=1 \\ \mu \neq \varkappa}}^{m} \mathfrak{F}_{\varkappa \mu}^{2} \bigcup\left(\mathfrak{c}_{\varkappa}^{3} \cap \mathfrak{b}_{0}^{3}\right)=\bigcap_{\chi_{\varkappa 1} \leqq \chi_{\varkappa} \leqq \chi_{\varkappa 2}} i_{\varkappa}^{1}\left(s_{\varkappa} e^{i \chi_{\varkappa}}\right)
$$

(2c) where

$$
\mathfrak{i}_{\varkappa_{1}}^{1}\left(s_{\varkappa} e^{i \chi_{\varkappa}}\right)=\bigcup_{\substack{\mu=1 \\ \mu \neq \varkappa}}^{m} \mathfrak{i}_{\varkappa \mu}^{1}\left(s_{\varkappa} e^{i \chi_{\varkappa}}\right) \bigcup \mathfrak{r}_{\varkappa}^{1}\left(s_{\varkappa} e^{i \chi_{\varkappa}}\right)
$$

is the boundary curve of $\mathfrak{\Im}_{\varkappa}^{2}\left(s_{\varkappa} e^{i \chi_{\varkappa}}\right)$; here

$$
\begin{gathered}
\dot{\mathfrak{i}}_{\varkappa \mu}^{1}\left(s_{\varkappa} e^{i \chi_{\varkappa}}\right)=\mathfrak{\Im}_{\varkappa}^{2}\left(s_{\varkappa} e^{i \chi_{\varkappa}}\right) \cap \mathfrak{e}_{\mu}^{3}, \quad \varkappa \neq \mu, \\
\mathfrak{r}_{\varkappa_{\varkappa}}^{1}\left(s_{\varkappa} e^{i \chi_{\varkappa}}\right)=\mathfrak{\Im}_{\varkappa}^{2}\left(s_{\varkappa} e^{i \chi_{\varkappa}}\right) \cap \mathfrak{b}_{0}^{3}
\end{gathered}
$$


2.3. The intersection $\mathfrak{I}^{2}(s)=\mathfrak{B}(s) \cap\left[z_{1}=0\right]$. Let

$$
\mathfrak{I}^{2}(s)=\left[z_{1}=0, z_{2}=p(s, \zeta)\right]=\mathfrak{S}(s) \bigcap\left[z_{1}=0\right]
$$

be a one-to-one image of $|\zeta| \leqq s$ onto $\mathfrak{I}^{2}(s), p(s, \zeta)$ is analytic in $\zeta$, and $O \in \mathfrak{I}^{2}(s)$ (3a). Its boundary $\dot{i}^{1}$, the image of $|\zeta|=s$, lies wholly in $\mathfrak{b}_{1}^{3}=\bigcup_{x=1}^{m} \mathfrak{e}_{\varkappa}^{3}(3 \mathrm{~b})$.

We assume that the intersection $\mathfrak{e}_{\varkappa}^{3} \cap\left[z_{1}=0\right]$ consists of one line segment

$$
\begin{gathered}
\dot{\mathfrak{j}}_{*}^{1}(s)=\mathfrak{e}_{*}^{3}(s) \cap\left[z_{1}=0\right]=\bigcup_{\chi_{\varkappa}=\chi_{\varkappa 3}}^{\chi_{\varkappa 4}}\left[\widetilde{\Im}_{\varkappa}^{2}\left(s_{\varkappa} e^{i \chi_{\varkappa}}\right) \cap\left[z_{1}=0\right]\right], \\
\chi_{\varkappa 1} \leqq \chi_{\varkappa 3} \leqq \chi_{\varkappa 4} \leqq \chi_{\varkappa 2},
\end{gathered}
$$

(3c). Here $\chi_{* 3}$ and $\chi_{* 4}$ are chosen so that

$$
\bigcup_{\chi_{\varkappa}=\chi_{\varkappa 3}}^{\chi_{\varkappa 4}} \mathfrak{J}_{\varkappa}^{2}\left(s_{\varkappa} e^{i \chi_{\varkappa}}\right)
$$

is the part of $\mathfrak{e}_{\varkappa}^{3}$ consisting of those laminas which include the points $\left(0, z_{2}\right)$ of $\mathfrak{T}^{2}$.

We assume that a lamina $\mathfrak{\Im}_{\varkappa}^{2}\left(s_{\varkappa} e^{i \chi_{\varkappa}}\right)$ contains at most one point $\left(0, z_{2}\right) \quad$ (3d). Let

$$
\mathfrak{j}^{1}(s)=\bigcup_{x=1}^{m} \mathfrak{j}_{*}^{1}(s)
$$

be 'an oriented differentiable curve in the $z_{2}$-plane $(3 \mathrm{e}) . \quad \dot{\mathfrak{j}}_{*}^{1}(s)$ consists of points $\left(0, z_{2}=s_{*} e^{i \chi_{*}}\right), \quad \chi_{* 3} \leqq \chi_{*} \leqq \chi_{* 4}$.

By

$$
w\left(s ; z_{2}\right), \quad w(s ; 0)=0,\left.\frac{d w\left(s ; z_{2}\right)}{d z_{2}}\right|_{z_{2}=0}>0
$$

we shall denote the function which maps $\mathfrak{I}^{2}(s)$ onto the disc $|\zeta|<s$ (i. e., $w\left(s ; z_{2}\right)$ is the inverse of $\left.z_{2}=p(s ; \zeta)\right)$.

\section{An integral formula for the value of $|f(0,0)|$ of a meromorphic function $f\left(z_{1}, z_{2}\right)$}

Let $f\left(z_{1}, z_{2}\right)$ be a meromorphic function in $\left[\left|z_{k}\right|<\infty, k=1,2\right]$, which is holomorphic at the origin. According to $(3 \mathrm{~b}), \quad \zeta=w\left(s ; z_{2}\right)$ $\operatorname{maps} \mathfrak{I}^{2}(s)$ onto $|\zeta| \leqq s$. 
By the Jensen formula

(1)

$$
\begin{gathered}
\log |f(0,0)|= \\
\frac{1}{2 \pi} \sum_{\varkappa=1}^{m} \int_{\mathrm{i}_{\varkappa}^{1}(s)} \log \left|f\left(0, s_{\varkappa} e^{i \chi_{\varkappa}}\right)\right| d \Omega\left(s ; s_{\varkappa} e^{i \chi_{\varkappa}}\right)+\sum_{k=1}^{2}(-1)^{k} \sum_{\mu} \log \left|\frac{s}{A_{k \mu}(s)}\right| .
\end{gathered}
$$

Here $A_{1_{\mu}}(s)$ are the images of the zeros of $f\left(0, z_{2}\right)$ in $|\zeta|<s$, and $A_{2 \mu}(s)$ are the images of the poles, $\Omega\left(s ; z_{2}\right)=\arg \left[w\left(s ; z_{2}\right)\right]$.

By $\left.(2.1)^{4}\right)$

$$
f\left[0, w\left(s ; s_{\varkappa} e^{i \chi_{\varkappa}}\right)\right]=f\left[h_{\varkappa}\left(s ; 0, s_{\varkappa} e^{i \chi_{\varkappa}}\right)\right]
$$

Thus

$$
\begin{gathered}
\log \left|f\left[h_{\varkappa}\left(s ; 0, s_{\varkappa} e^{i \chi_{\varkappa}}\right)\right]\right|= \\
\frac{1}{2 \pi} \int_{\varphi=0}^{2 \pi} \log \left|f\left[h_{\varkappa}\left(s ; r e^{i \varphi}, s_{\varkappa} e^{i \chi_{\varkappa}}\right)\right]\right| d \varphi+\sum_{k=1}^{2}(-1)^{k} \sum_{\mu} \log \mid \frac{r}{a_{k \mu}\left(s_{\varkappa} e^{i \chi_{\varkappa}}\right)} .
\end{gathered}
$$

The $a_{k \mu}\left(s_{\varkappa} e^{i \chi_{k}}\right)$ are the zeros $(k=1)$ and poles $(k=2)$ of $f\left[h_{k}\left(s ; \hat{\mathbf{Z}}_{\varkappa}, s_{k} e^{i \chi_{\varkappa}}\right)\right]$ in $\left|\hat{\mathbf{Z}}_{\varkappa}\right| \leqq r$.

Combining (1), (2) and (3), we obtain ${ }^{5}$ )

$$
\begin{aligned}
& \log |f(0,0)|= \\
& \frac{1}{4 \pi^{2}} \sum_{\varkappa=1}^{m} \int_{\chi_{\varkappa 3}}^{\chi_{\varkappa 4}} \int_{\varphi=0}^{2 \pi} \log \mid f\left[h_{\varkappa}\left(s ; r(s) e^{i \varphi}, s_{\varkappa} e^{\left.i \chi_{\varkappa}\right)}\right] \mid \frac{d \Omega\left(s ; s_{\varkappa} e^{i \chi_{\varkappa}}\right)}{d \chi_{\varkappa}} d \chi_{\varkappa} d \varphi\right. \\
& +\frac{1}{2 \pi} \sum_{k=1}^{2}(-1)^{k} \sum_{\mu} \sum_{\varkappa=1}^{m} \int_{\chi_{\varkappa 3}}^{\chi_{\varkappa 4}} \log \frac{r(s)}{\mid a_{k \mu}\left(s_{\varkappa} e^{\left.i \chi_{\varkappa}\right) \mid} \frac{d \Omega\left(s ; s_{\varkappa} e^{i \chi_{\varkappa}}\right)}{d \chi_{\varkappa}} d \chi_{\varkappa}\right.} \\
& +\sum_{k=1}^{2}(-1)^{k} \sum_{\mu} \log \frac{s}{\left|A_{k \mu}(s)\right|} .
\end{aligned}
$$

Following the Nevanlinna procedure [21]-[22], we write (4) in the form

$$
\begin{aligned}
& \log |f(0,0)|+\mathbf{m}\left(\mathfrak{F}^{2}(s), f^{-1}\right)+\tilde{\mathbf{N}}\left(\mathfrak{F}^{2}(s), f^{-1}\right)+P\left(s, f^{-1}\right) \\
& =\mathbf{m}\left(\mathfrak{F}^{2}(s), f\right)+\tilde{\mathbf{N}}\left(\mathfrak{F}^{2}(s), f\right)+P(s, f) \equiv \mathbf{T}\left(\mathfrak{\mho}^{2}(s), f^{\prime}\right),
\end{aligned}
$$

4) For brevity's sake we shall write $f\left[h_{x}\left(s ; \hat{Z}, s_{\varkappa_{x}} \exp \left(i \chi_{x}\right)\right)\right]$ instead of $f\left[h_{1 \varkappa}\left(s ; \hat{Z}, s_{\varkappa} \exp \left(i \chi_{\varkappa}\right)\right), h_{2 \varkappa}\left(s ; \hat{Z}, s_{\varkappa} \exp \left(i \chi_{\varkappa}\right)\right)\right]$.

5 ) It should be noted that in the formulas (4.2) and (4.3), p. 33, of [12] the factors $1 /\left(4 \pi^{2}\right)$ and $1 /(2 \pi)$, respectively, are missing. 
where

(6)

$$
\begin{gathered}
\mathrm{m}\left(\mathfrak{F}^{2}(s), f\right)= \\
\frac{1}{4 \pi^{2}} \sum_{\varkappa=1}^{m} \int_{\chi_{\varkappa 3}}^{\chi_{\varkappa 4}} \int_{\varphi=0}^{2 \pi} \log \left|f\left[h_{\varkappa}\left(s ; r(s) e^{i \varphi}, s_{\varkappa} e^{i \chi_{\varkappa}}\right)\right]\right| \frac{d \Omega\left(s ; s_{\varkappa} e^{i \chi_{\varkappa}}\right)}{d \chi_{\varkappa}} d \chi_{\varkappa} d \varphi, \\
\tilde{\mathbf{N}}\left(\mathfrak{F}^{2}(s), f\right)=\frac{1}{2 \pi} \int_{\chi_{\varkappa 3}}^{+} \sum_{\mu} \log \left|\frac{r(s)}{a_{2 \mu}\left(s_{\varkappa} e^{i \chi_{\varkappa}}\right)}\right| \frac{d \Omega\left(s ; s_{\varkappa} e^{i \chi_{\varkappa}}\right)}{d \chi_{\varkappa}} d \chi_{\varkappa}, \\
P(s, f)=\sum_{\mu} \log \left|\frac{s}{A_{2 \mu}(s)}\right|
\end{gathered}
$$

Remark 2. It should be noted that

(9) $\quad \mathbf{m}\left(\mathfrak{F}^{2}(s), f\right)=\frac{1}{2 \pi} \sum_{\varkappa=1}^{m} \int_{\chi_{\varkappa 2}}^{\chi_{\varkappa 3}} m\left[r(s), f\left(h_{\varkappa}\left(s ; \hat{\mathrm{Z}}, s_{\varkappa} e^{i \chi_{\varkappa}}\right)\right)\right] \frac{d \Omega\left(s ; s_{\varkappa} e^{i \chi_{\varkappa}}\right)}{d \chi_{\varkappa}} d \chi_{\varkappa}$,

(10) $\tilde{\mathbf{N}}\left(\widetilde{F}^{2}(s), f\right)=\frac{1}{2 \pi} \sum_{\varkappa=1}^{m} \int_{\chi_{\varkappa 2}}^{\chi_{\varkappa 3}} N\left[r, f\left(h_{\varkappa}\left(s ; \hat{\mathbf{Z}}, s_{\varkappa} e^{i \chi_{\varkappa}}\right)\right)\right] \frac{d \Omega\left(s ; s_{\varkappa} e^{i \chi_{\varkappa}}\right)}{d \chi_{\varkappa}} d \chi_{\varkappa}$,

$$
P(s, f)=N[s ; f(0, w(s ; \zeta))] .
$$

Repeating the usual considerations one obtains the analogue of the first main inequality.

Theorem 3.1. Let $f\left(z_{1}, z_{2}\right)$ be a meromorphic function of two complex variables which is regular at the origin. Then

$$
\begin{gathered}
\mathbf{m}\left(\mathfrak{F}^{2}(s), \frac{1}{f-\alpha}\right)+\tilde{\mathbf{N}}\left(\mathfrak{F}^{2}(s), \frac{1}{f-\alpha}\right)+P\left(s, \frac{1}{f-\alpha}\right)=\mathbf{T}\left(\mathfrak{F}^{2}(s), f\right)+h(r, s), \\
f(0,0) \neq \alpha,
\end{gathered}
$$

where

$$
|h(r, s)| \leqq \stackrel{+}{\log }|\alpha|+\log 2 .
$$

If $f(0,0)-\alpha=0$, the formula (12) has to be slightly modified.

Since the proof is exactly the same as in the case of one variable, we do not repeat it.

Until present $s, s_{\varkappa}, \varkappa=1,2, \ldots, m$, and $r$ have been some fixed quantities. From now on we shall assume that

$$
s_{\varkappa}=s_{\varkappa}(s), r=R(s)\left(\lim _{s \rightarrow \infty} \frac{R(s)}{s^{\mu}}=A<\infty, 0<\mu<\infty, A>0\right)
$$


are continuous, increasing functions of $s$; the domains $\mathfrak{B}(s)$, $s_{0} \leqq s<\infty$, have the property that $\mathfrak{B}\left(s_{2}\right) \supset \mathfrak{B}\left(s_{1}\right)$ for $s_{2}>s_{1}$. Further we assume that the distinguished boundary surfaces $\mathfrak{F}^{2}(s)$ change continuously, forming a three-dimensional manifold

$$
\tilde{\mathfrak{r}}^{3}=\bigcup_{s=s_{0}}^{\infty} \mathfrak{F}^{2}(s)
$$

$\mathbf{m}\left(\mathfrak{F}^{2}(s), f\right)$ is a functional which represents a measure for the growth of $\log |f|$ on $\tilde{r}^{3}$ for $s \rightarrow \infty$.

$$
\mathfrak{v}=\bigcup_{s=s_{0}}^{\infty} \bigcup_{\varkappa=0}^{m} \mathfrak{e}_{\varkappa}^{3}(s)
$$

is a four-dimensional domain in the $z_{1}, z_{2}$-space. If we assume that

$$
\left(\bigcup_{x=1}^{m} \mathfrak{e}_{\kappa}^{3}\left(s_{1}\right)\right) \cap\left(\bigcup_{x=1}^{m} \mathfrak{e}_{x}^{3}\left(s_{2}\right)\right)=\varnothing \quad \text { for } \quad s_{1} \neq s_{2}
$$

$\tilde{\mathbf{N}}\left(\mathfrak{F}^{2}(s), f\right)$ can be considered as a functional "measuring» the density of pole-surfaces in $\mathfrak{\boldsymbol { E }}$.

Finally, $P(s, f)$ is a functional connected with the number of poles in the domain

$$
z_{1}=0, \quad 0<\left|z_{2}\right|<\infty \text {. }
$$

Repeating the usual considerations, we obtain the relation

$$
\begin{gathered}
\mathbf{m}\left(\mathfrak{F}^{2}(s),(f-a)^{-1}\right)+\tilde{\mathbf{N}}\left(\mathfrak{F}^{2}(s),(f-a)^{-1}\right)+P\left(s,(f-a)^{-1}\right) \\
=\mathbf{T}\left(\mathfrak{F}^{2}(s), f\right)+h(r(s), a), \\
\mathbf{T}\left(\mathfrak{F}^{2}(s), f\right)=\mathbf{m}\left(\mathfrak{F}^{2}(s), f\right)+\widetilde{\mathbf{N}}\left(\mathfrak{F}^{2}(s), f\right)+P(s, f), \\
|h(r(s), a)| \leqq \log a+\log 2,
\end{gathered}
$$

an analogue of the first Nevanlinna theorem.

Remark 3. It should be noted that in the case $m=1$ it is possible to show that the growth of $\tilde{\mathbf{N}}\left(\mathfrak{F}^{2}(s), f\right)$ is essentially the same as the growth of a functional which measures the density of lines

$$
\left[f\left(z_{1}, z_{2}\right)=a\right] \cap \bigcup_{\sigma=s_{0}}^{s} \mathfrak{F}^{2}(\sigma), \quad a=\text { complex constant } .
$$

In Section 4 an analogue of the second Nevanlinna theorem will be considered. 


\section{An analogue of the second Nevanlinna theorem}

In this section we assume that $m=1$. We set $s_{1}=s, \chi_{1}=\chi$. In addition of considering the functional $\mathbf{m}\left(\mathfrak{F}^{2}(s), f\right)$ (a »measure» of the growth of $|f|$ on $\left.\tilde{i}^{3}=\bigcup \mathfrak{F}^{2}(s)\right)$ and $\tilde{\mathbf{N}}\left(\mathfrak{F}^{2}(s), f\right)$ which measures the density of pole surfaces in the domain $\mathbb{E}$ (bounded by $\tilde{\mathbf{T}}^{3}$ ), we can consider the analogous measure in three-dimensional sets lying inside $\mathfrak{E}$. A set of this kind will be introduced in the following:

Let $h_{k 1}\left(s ; \hat{\mathbf{Z}}_{k}, s e^{i \chi}\right)$ be again the function pair $(2.1)$ and $\mathfrak{\Im}_{1}^{2}\left(s e^{i \chi}\right)$ be the lamina (2.2). By $\Re_{1}^{2}\left(s e^{i \chi}\right)$ we denote a subdomain of $\mathfrak{\Im}_{1}^{2}\left(s e^{i \chi}\right)$, which lies inside of $\mathfrak{\Im}_{1}^{2}\left(s e^{i x}\right)$ and is bounded by

(1) $\tilde{\mathfrak{r}}_{1}^{1}\left(s e^{i \chi}\right)=\left[z_{k}=h_{k 1}\left(s ; \tilde{r}\left(s e^{i \chi}\right) e^{i \varphi}, s e^{i \chi}\right), 0 \leqq \varphi \leqq 2 \pi\right], \quad 0 \leqq \chi \leqq 2 \pi$,

$$
\tilde{r}\left(s e^{i \chi}\right) \equiv \tilde{r}\left(s ; s e^{i \chi}\right),
$$

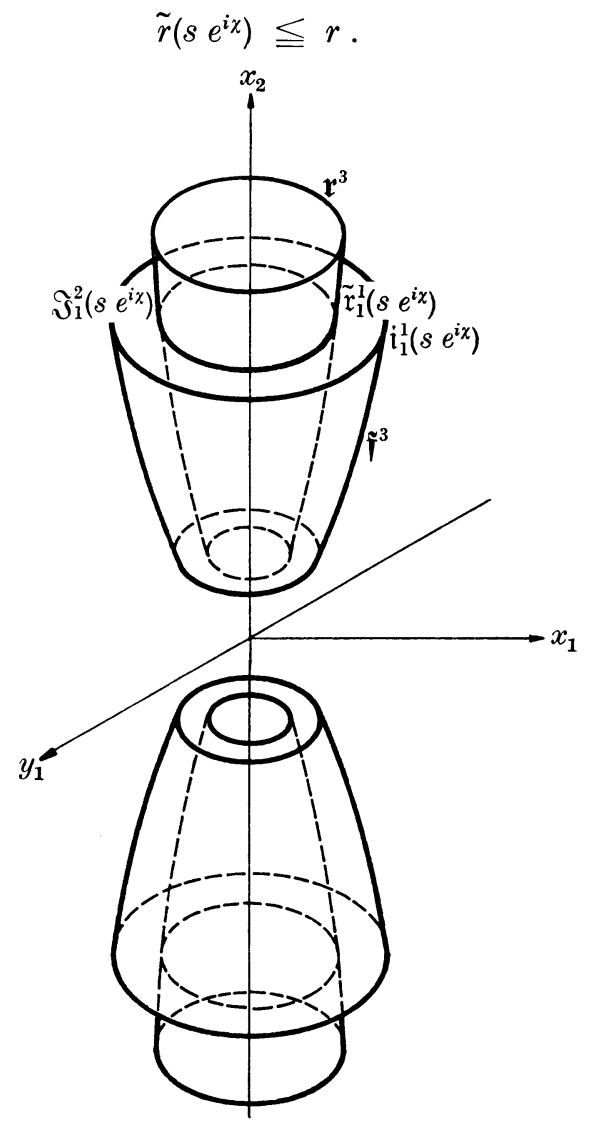

Fig. 1

The intersection of $\tilde{\boldsymbol{r}}^{3}$ and $\mathfrak{r}^{3}$ with the hyperplane $y_{2}=0$ 
Definition. The integral

$$
\int_{\chi=0}^{2 \pi}+\log \left[\frac{1}{r\left(s e^{i \chi}\right)-\tilde{r}\left(s e^{i \chi}\right)}\right] \frac{d \Omega\left(s ; s e^{i \chi}\right)}{d \chi} d \chi
$$

will be called the pseudo-distance between $\mathfrak{i}_{1}^{1}\left(s e^{i \chi}\right)$ and $\tilde{\mathfrak{r}}_{1}^{1}\left(s e^{i \chi}\right)$ and is denoted by

$$
\mathrm{P}\left(\mathfrak{F}_{1}^{2}(s), \mathfrak{R}_{1}^{2}(s)\right)
$$

Here

$$
\Re_{1}^{2}(s)=\bigcup_{\chi=0}^{2 \pi} \tilde{\mathfrak{r}}_{1}^{1}\left(s e^{i \chi}\right) .
$$

Concerning $\mathfrak{F}_{1}^{2}(s)$, see $(2.8)$.

Let $f\left(z_{1}, z_{2}\right)$ be a meromorphic function of two complex variables in $\left[\left|z_{k}\right|<\infty, k=1,2\right]$, which has the following property: The function $f\left[h_{1}\left(s ; \hat{\mathrm{Z}}, s e^{i \chi}\right)\right]$, see (2.1), has the development

$$
f\left[h_{1}\left(s ; \hat{\mathbf{Z}}, s e^{i \chi}\right)\right]=c_{0}\left(s e^{i \chi}\right)+c_{1}\left(s e^{i \chi}\right) \hat{\mathbf{Z}}+\ldots, \quad|\hat{\mathbf{Z}}| \leqq r .
$$

Except in an at most denumerable set of points $(s, \chi), \quad c_{0}\left(s e^{i \chi}\right) \neq 0$ and $c_{1}\left(s e^{i \chi}\right)$ have the following properties:

$$
\int_{\chi=0}^{2 \pi}+\frac{1}{\log \frac{1}{\left|c_{1}\left(s e^{i \chi}\right)\right|}} \frac{d \Omega\left(s ; s e^{i \chi}\right)}{d \chi} d \chi \leqq A \log s, \quad 0<s_{0} \leqq s<\infty
$$

where $A<\infty$ is a positive constant (4a).

Let $\alpha_{v}, v=1,2, \ldots, q$, be $q$ constants different from each other and let

$$
\begin{aligned}
& R_{0}\left(s e^{i \chi}\right)=\max _{\nu=1,2, \ldots, q}\left[\frac{1}{\left|c_{0}\left(s e^{i \chi}\right)-\alpha_{\nu}\right|}\right], \\
& c_{0}\left(s e^{i \chi}\right) \equiv f\left[h_{1}\left(s ; 0, s e^{i \chi}\right)\right] .
\end{aligned}
$$

We assume that

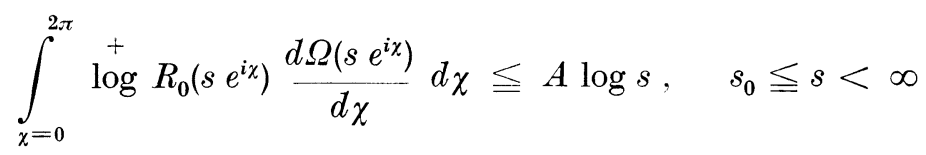

(4b). We assume that for $f\left(z_{1}, z_{2}\right)$

(10) $T\left[r, f\left[h_{1}\left(s ; \hat{\mathrm{Z}}, s e^{i \chi}\right)\right]\right] \equiv T\left[r, f\left[h_{11}\left(s ; \hat{\mathrm{Z}}, s e^{i \chi}\right), h_{12}\left(s ; \hat{\mathbf{Z}}, s e^{i \chi}\right)\right]\right]$

$$
\leqq A r^{\lambda_{1}} s^{\lambda_{2}}
$$


holds for $0 \leqq \lambda_{k}<\infty, A<\infty$, where

$$
s=S(r)
$$

and $S(r)$ is the function inverse to $R(s)$, see (3.14) (4c).

Theorem 4.1. Let

$$
\mathfrak{r}^{3}=\bigcup_{s=s_{0}}^{\infty} \Re_{1}^{2}(s)
$$

where $\Re_{1}^{2}(s)$ is the surface given by (5). Let

$$
\mathrm{P}\left(\mathfrak{F}_{1}^{2}(s), \mathfrak{R}_{1}^{2}(s)\right) \leqq A \log s, \quad A<\infty .
$$

Let $\alpha_{v}, \quad v=1,2, \ldots, q$, be $q$ constants different from each other;

We assume further that $f\left(z_{1}, z_{2}\right)$ is a meromorphic function of two complex variables regular at the origin, with the development (6) in the lamina $\mathfrak{\Im}^{2}\left(s e^{i \chi}\right)$. Here the coefficients $c_{0}\left(s e^{i \chi}\right)$ and $c_{1}\left(s e^{i \chi}\right)$ satisfy the inequatities (7) and (9).

In addition, let the characteristic functions $T\left[r, f\left[h_{1}\left(s ; \hat{\mathrm{Z}}, s e^{i \chi}\right)\right]\right]$ satisfy the inequality (10). Then

(14) $\quad(q-2) \mathbf{T}\left(\Re_{1}^{2}(s), f\right) \leqq \sum_{\nu=1}^{q} \mathbf{N}\left(\Re_{1}^{2}(s),\left(f-\alpha_{v}\right)^{-1}\right)+A \log s, \quad A<\infty$.

Proof. We consider $f\left[h_{1}\left(s ; \hat{\mathrm{Z}}_{1}, s e^{i \chi}\right)\right]$ for fixed $s, \chi$ as a function of the complex variable $\hat{\mathrm{Z}}_{1}$. $\mathrm{By}\left(\mathrm{II}^{\prime}\right)$, p. 69, and $(20)$, p. 68, of [21] it holds

$$
\begin{aligned}
& (q-2) T\left[\tilde{r}\left(s e^{i \chi}\right), f\left[h_{1}\left(s ; \hat{\mathrm{Z}}_{1}, s e^{i \chi}\right)\right]\right] \\
& \leqq \\
& \sum_{\nu=1}^{q} N\left[\tilde{r}\left(s e^{i \chi}\right),\left[f\left[h_{1}\left(s ; \hat{\mathrm{Z}}_{1}, s e^{i \chi}\right)\right]-\alpha_{\nu}\right]^{-1}\right] \\
& \quad+56+\underset{+}{+} \frac{1}{\left|c_{1}\left(s e^{i \chi}\right)\right|}+6 q \log ^{+} R_{0}\left(s e^{i \chi}\right)+4+\log ^{+} \log R \\
& \quad+2 q \log \frac{2 q}{\delta}+4 \log ^{+} \frac{1}{\left|\tilde{r}\left(s ; s e^{i \chi}\right)\right|}+8 \log \left|r\left(s ; s e^{i \chi}\right)\right| \\
& \quad+6 \log \frac{1}{r\left(s ; s e^{i \chi}\right)-\tilde{r}\left(s ; s e^{i \chi}\right)} \\
& \quad+\quad+\log T\left[r\left(s ; s e^{i \chi}\right), f\left[h_{1}\left(s ; \hat{Z}_{1}, s e^{i \chi}\right)\right]\right] .
\end{aligned}
$$

Here

$$
R=\max _{\nu=1,2, \ldots, q}\left(\left|\alpha_{\nu}\right|\right) \text { and } \delta=\min _{\substack{\nu \neq \mu \\ \nu, \mu=1,2, \ldots, q}}\left(1,\left|\alpha_{\nu}-\alpha_{\mu}\right|\right)
$$


We apply the operation

$$
\frac{1}{2 \pi} \int_{\chi=0}^{2 \pi} \ldots \frac{d \Omega\left(s ; s e^{i \chi}\right)}{d \chi} d \chi
$$

to (15). In accordance with our hypotheses this operation is admissible for $s_{0} \leqq s<\infty$. Using (3.9) and (3.10), we obtain that the left-hand side becomes the left-hand side of (16); further, the first term becomes the first term of the right-hand side of (16). Thus,

$$
\begin{aligned}
& (q-2)\left[\mathbf{T}\left(\Re_{1}^{2}(s), f\right)-P(s, f)\right] \\
& \leqq \sum_{\nu=1}^{q} \hat{\mathbf{N}}\left(\Re_{1}^{2}(s),\left(f-\alpha_{\nu}\right)^{-1}\right)+\frac{1}{2 \pi} \int_{\chi_{1}=0}^{2 \pi} \log \frac{1}{\left|c_{1}\left(s e^{i \chi}\right)\right|} \frac{d \Omega}{d \chi} d \chi \\
& +\frac{9}{\pi} \int_{\chi=0}^{2 \pi}+\stackrel{+}{\log } R_{0}\left(s e^{i \chi}\right) \frac{d \Omega}{d \chi} d \chi+\frac{2}{\pi}+\stackrel{+}{\log } \log R \int_{0}^{2 \pi} \frac{d \Omega}{d \chi} d \chi \\
& +\left(56+\frac{1}{\pi} q \log \frac{2 q}{\delta}\right) \int_{\chi=0}^{2 \pi} \frac{d \Omega}{d \chi} d \chi+\frac{2}{\pi} \int_{\chi=0}^{2 \pi} \log \frac{1}{\left|\tilde{r}\left(s ; s e^{i \chi}\right)\right|} \frac{d \Omega}{d \chi} d \chi \\
& +\frac{4}{\pi} \int_{\chi_{1}=0}^{2 \pi} \log \left|r\left(s ; s e^{i \chi}\right)\right| \frac{d \Omega}{d \chi} d \chi+\frac{3}{\pi} \int_{\chi=0}^{2 \pi}+\log \frac{1}{r\left(s ; s e^{i \chi}\right)-\tilde{r}\left(s ; s e^{i \chi}\right)} \frac{d \Omega}{d \chi} d \chi \\
& +\frac{4}{\pi} \int_{\chi=0}^{2 \pi} \log T\left[r\left(s ; s e^{i \chi}\right), f\left[h_{1}\left(s ; \hat{\mathbf{Z}}_{1}, s e^{i \chi}\right)\right]\right] \frac{d \Omega}{d \chi} d \chi, \\
& \frac{d \Omega}{d \chi} \equiv \frac{d \Omega\left(s ; s e^{i \chi}\right)}{d \chi}
\end{aligned}
$$

According to hypothesis (3a), the function $\quad \zeta=w\left(s ; z_{2}\right)$ maps the (simply connected domain) $\mathfrak{I}^{2}(s)$ onto the disc $|\zeta|<s$, see 2.3 p. 7 . According to our assumptions $\arg \zeta=\Omega$. If $\zeta$ varies along the circle in the $\zeta$-plane, its image in the $z_{2}=0$ plane varies along the boundary of $\mathfrak{I}^{2}(s)$. Since $\mathfrak{I}^{2}(s)$ contains the origin and the mapping is one-to-one, it follows that

$$
\int_{\chi=0}^{2 \pi} \frac{d \Omega\left(s ; s e^{i \chi}\right)}{d \chi} d \chi=2 \pi
$$


Therefore, the fourth and fifth terms are constant. By (7) and (9) the second and third terms, respectively, are majorized by $A \log s$. By (3), (3.14) and (17) the sixth term is majorized by a constant, while the seventh term is smaller than $A \log s$. By (4) and (13) the eight term is smaller than $A \log s$. Finally, by (10) the same holds for the last term of the right-hand side of (16).

\section{Bounds for holomorphic functions of special classes}

The present section will deal with the second approach in the study of functions of two complex variables, especially the application of the Schottky inequality and certain theorems on schlicht functions in the theory of functions of one complex variable.

Through every point of the $z_{1}, z_{2}$-space, except the origin, passes one and only one plane

$$
\mathfrak{P}^{2}(\alpha)=\left[z_{2}=\alpha z_{1}\right], \quad \mathfrak{P}^{2}(\infty)=\left[z_{1}=0\right] .
$$

Remark 1. Through every point $x_{1}^{(0)}, x_{2}^{(0)}, y_{2}^{(0)}, x_{1}^{(0)}>0$, of $\left[x_{1}^{2}+x_{2}^{2}\right.$ $\left.+y_{2}^{2}=1, x_{1}>0, y_{1}=0\right]$ passes one straight line $\mathfrak{P}^{2}(\alpha) \cap\left[y_{1}=0\right]$, $\alpha=\left(x_{2}^{(0)}+i y_{2}^{(0)}\right) / x_{1}^{(0)}$. The intersection of every plane $\mathfrak{P}^{2}(\alpha), \alpha \neq 0$, with $y_{1}=y_{1}^{0} \neq 0$, is a straight line, $x_{2}=\alpha_{1} x_{1}-\alpha_{2} y_{1}^{0}, y_{2}=\alpha_{2} x_{1}+$ $\alpha_{1} y_{1}^{0}, \quad \alpha=\alpha_{1}+i \alpha_{2}$.

Let $\mathfrak{\Omega}^{2}$ be a simply connected domain in the plane, $O \in \mathfrak{\Omega}^{2}, \infty \notin \overline{\mathfrak{\Omega}^{2}}$, and let $\mathfrak{q}^{1}$, the boundary of $\mathfrak{\Omega}^{2}$, be a differentiable curve of finite length.

Let

$$
\mathfrak{D}^{3}=\bigcup_{\chi=0}^{2 \pi}\left[\mathfrak{P}^{2}\left(s e^{i \chi}\right),\left|z_{1}\right| \leqq r\right], \quad \mathfrak{P}^{2}\left(s e^{i \chi}\right) \equiv\left[z_{2}=s e^{i \chi} z_{1}\right] .
$$

The domain

$$
\mathscr{D}=\left[\bigcup_{|\alpha| \leqq s} \mathfrak{P}^{2}(\alpha)\right] \cap\left[\left|z_{1}\right| \leqq r\right],
$$

to be considered here, is bounded by the segments of analytic hypersurfaces $\grave{D}^{3}$ and

$$
\mathfrak{b}^{3}=\bigcup_{\varphi=0}^{2 \pi}\left[z_{1}=r e^{i \varphi}\right] \cap\left[\left|z_{2}\right| \leqq s r\right],
$$

see Fig. $\left.2^{6}\right)$, pp. 16 and 17.

$\left.{ }^{6}\right)$ The straight lines marked by $1,2,3,4,5$, and 6 correspond to the intersections of $\left[\mathfrak{P}^{2}(\alpha),\left|z_{1}\right|<r\right]$ with $y_{1}=0$ for $\alpha=i, 1,(4-i) / \sqrt{17},-i,-1$, $(-4+i) / \sqrt{17}$, respectively. In Fig. $2 \mathrm{~b}$ they are intersections with $y_{1}=1 / 4$. 


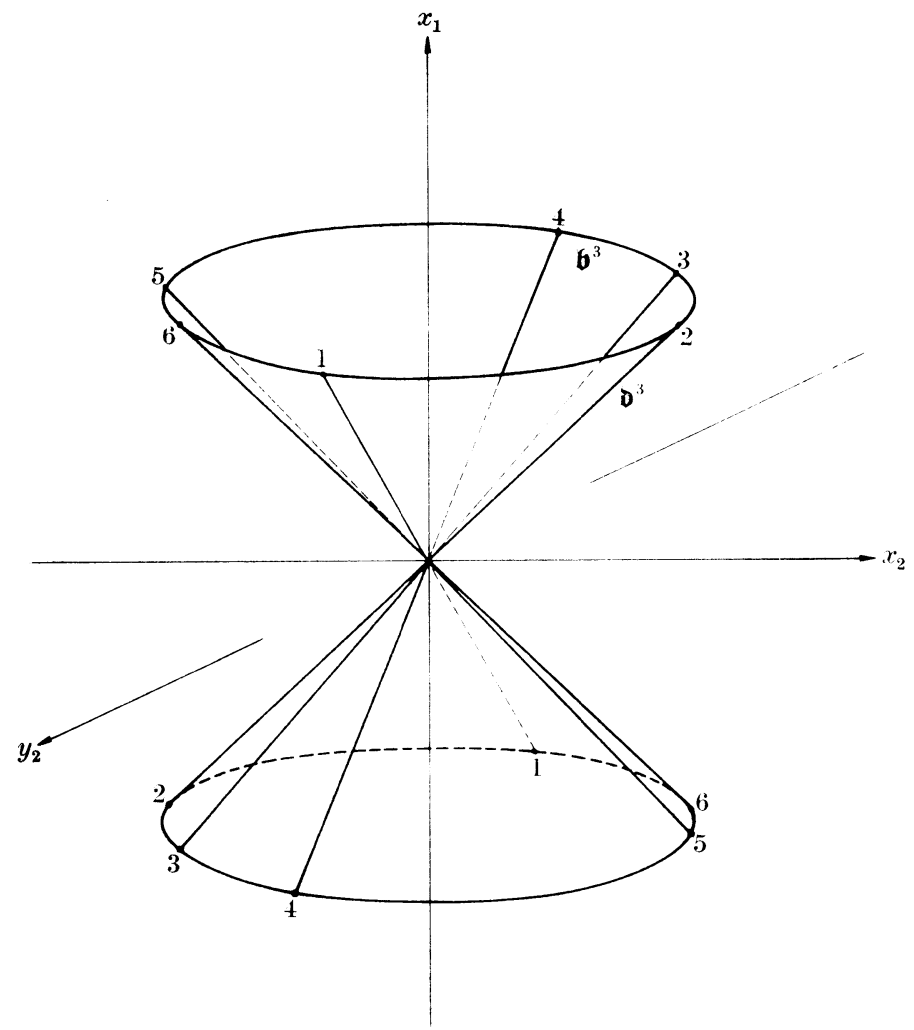

Fig. 2a

The intersection of 2 with the hyperplane $y_{1}=0$

Theorem 5.1. Let $f\left(z_{1}, z_{2}\right)$ be an analytic function of two complex variables, $z_{1}, z_{2}$, holomorphic in $\overline{\mathfrak{D}} ; f\left(z_{1}, z_{2}\right)$ omits in every $\left[\mathfrak{P}^{2}\left(s e^{i \chi}\right)\right.$, $\left.\left|z_{1}\right| \leqq r\right]$ the values $A_{1}(\chi)$ and $A_{2}(\chi), A_{1}(\chi) \neq A_{2}(\chi)$, and has the development

$$
f\left(z_{1}, z_{2}\right)=a_{00}+a_{10} z_{1}+a_{01} z_{2}+\ldots
$$

at the origin. Then the inequality

$$
\begin{gathered}
\log \left|f\left(z_{1}, z_{2}\right)\right|+\sum_{\nu} \log \mid \frac{\left(s\left|z_{1}\right|\right)^{2}-\overline{n_{\nu}\left(z_{1}\right)} z_{2} \mid}{s\left|z_{1}\right|\left(z_{2}-n_{\nu}\left(z_{1}\right)\right)} \\
\leqq \\
\frac{1}{2 \pi} \int_{0}^{2 \pi}\left\{\frac{r+\left|z_{1}\right|}{r-\left|z_{1}\right|}\left[\log \left|\frac{a_{00}-A_{1}(\chi)}{A_{2}(\chi)-A_{1}(\chi)}\right|+7\right]+\log \left|A_{2}(\chi)-A_{1}(\chi)\right|\right. \\
\left.+\log ^{+}\left|A_{1}(\chi)\right|+\log 2\right\} \frac{\left(s^{2}\left|z_{1}\right|^{2}-\left|z_{2}\right|^{2}\right) d \chi}{\left|s z_{1}\right|^{2}+\left|z_{2}\right|^{2}-2 s\left|z_{1} z_{2}\right| \cos \left(\chi-\arg z_{2}\right)}
\end{gathered}
$$




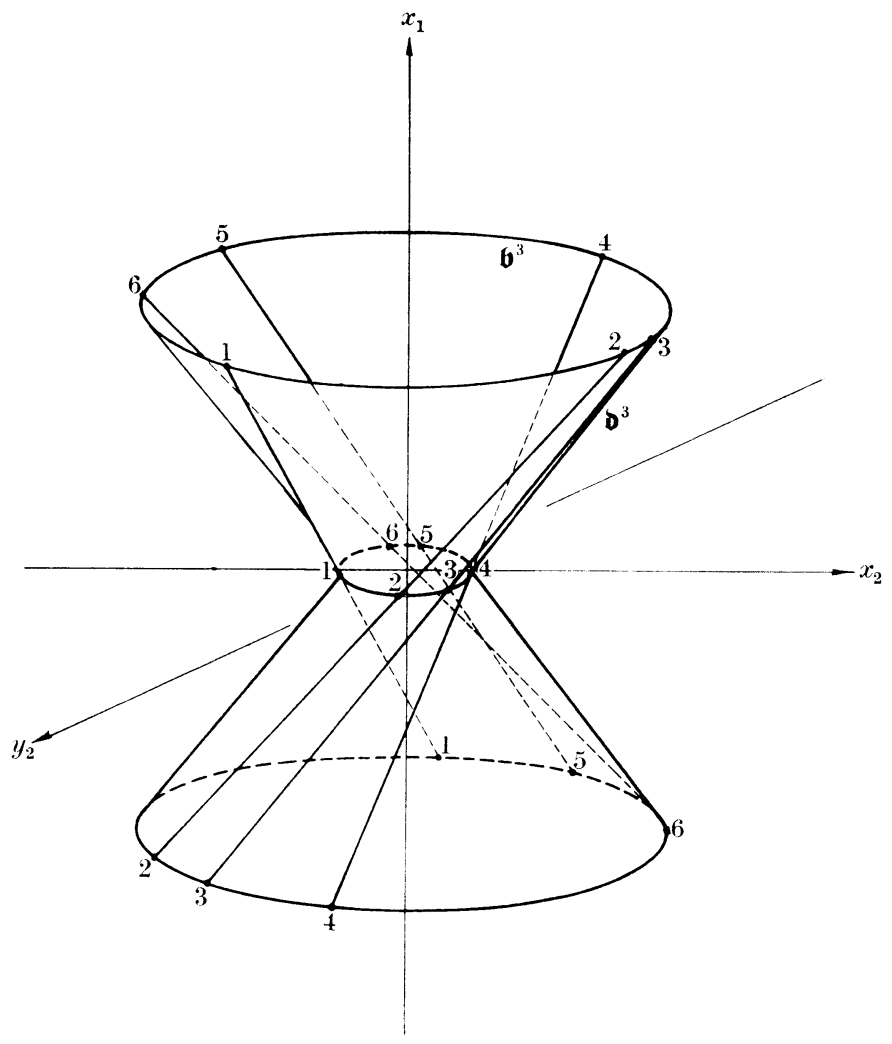

Fig. $2 b$

The intersection of 2 with the hyperplane $y_{1}=\frac{1}{4}$

holds for $f\left(z_{1}, z_{2}\right)$, when $\left(z_{1}, z_{2}\right) \in \mathfrak{2}$. Here $n_{v}\left(z_{1}\right)$ are the zero points of $f\left(z_{1}, z_{2}\right)$ in $\left|z_{2}\right| \leqq s\left|z_{1}\right|$.

Proof. We consider first the function $f\left(z_{1}, z_{2}\right)$ in the lamina $\left[\mathfrak{P}^{2}\left(s e^{i \chi}\right)\right.$, $\left.\left|z_{1}\right| \leqq r\right], \quad$ i. e., the function

$$
F_{\chi}\left(z_{1}\right) \equiv f\left(z_{1}, s e^{i \chi} z_{1}\right)=a_{00}+\left(a_{10}+s e^{i \chi} a_{01}\right) z_{1}+\ldots
$$

in the disc $\left|z_{1}\right| \leqq r$. Since $F_{\chi}\left(z_{1}\right)$ omits the values $A_{1}(\chi)$ and $A_{2}(\chi)$ in $\left|z_{1}\right| \leqq r$,

$$
G_{\chi}\left(z_{1}\right) \equiv \frac{F_{\chi}\left(z_{1}\right)-A_{1}(\chi)}{A_{2}(\chi)-A_{1}(\chi)}, \quad\left|A_{1}(\chi)\right| \leqq\left|A_{2}(\chi)\right|,
$$

omits the values 0 and 1 in $\left|z_{1}\right| \leqq r$.

Applying the Schottky theorem and using the Ahlfors inequality [1], [18] p. 294, we have 
(8)

$$
\begin{gathered}
\log \left|F_{\chi}\left(z_{1}\right)-A_{1}(\chi)\right| \leqq \\
\frac{r+\left|z_{1}\right|}{r-\left|z_{1}\right|}\left\{\stackrel{+}{\log }\left|G_{\chi}(0)\right|+7\right\}+\log \left|A_{2}(\chi)-A_{1}(\chi)\right|, \quad\left|z_{1}\right|<r .
\end{gathered}
$$

Consequently,

(9) $\left|F_{\chi}\left(z_{1}\right)\right| \leqq\left|A_{2}(\chi)-A_{1}(\chi)\right| \exp \left[\frac{r+\left|z_{1}\right|}{r-\left|z_{1}\right|}\left(\stackrel{+}{\log }\left|G_{\chi}(0)\right|+7\right)\right]+\left|A_{1}(\chi)\right| \cdot$

Since

$$
\frac{r+\left|z_{1}\right|}{r-\left|z_{1}\right|}\left(\log \left|G_{x}(0)\right|+7\right)>1
$$

it holds

$$
\stackrel{+}{\log }\left|f\left(z_{1}, s e^{i \chi} z_{1}\right)\right| \equiv \stackrel{+}{\log }\left|F_{\chi}\left(z_{1}\right)\right|
$$

$\leqq \frac{r+\left|z_{1}\right|}{r-\left|z_{1}\right|}\left[\log \left|G_{\chi}(0)\right|+7\right]+\stackrel{+}{\log }\left|A_{2}(\chi)-A_{1}(\chi)\right|+\stackrel{+}{\log }\left|A_{1}(\chi)\right|+\log 2$.

The intersection

$$
\text { จ } \cap\left[z_{1}=r_{1} e^{i \varphi}\right], \quad r_{1} e^{i \varphi}=z_{1}^{0}, \quad r_{1}<r,
$$

is the disc $\mathfrak{C}^{2}=\left[\left|z_{2}\right| \leqq r_{1} s\right]$. In $\mathfrak{C}^{2}$ the function $f\left(z_{1}, z_{2}\right)$ becomes

$$
H\left(z_{2}\right) \equiv f\left(r_{1} e^{i \varphi}, z_{2}\right), \quad\left|z_{2}\right| \leqq r_{1} s ;
$$

it holds

$$
H\left(r_{1} s e^{i \chi}\right)=f\left(r_{1} e^{i \varphi}, r_{1} s e^{i \chi}\right) \equiv F_{\chi}\left(r_{1} e^{i \varphi}\right) .
$$

The function $H\left(z_{2}\right)$ is a holomorphic function of a complex variable which, at the boundary point $z_{2}=r_{1} s e^{i x}$, assumes values for which we obtained the upper bound (10). Applying the inequality of PoissonJensen yields

$$
\begin{gathered}
\leqq \frac{1}{2 \pi} \int_{0}^{2 \cdot \tau}\left\{\frac{r+\left|z_{1}^{0}\right|}{r-\left|z_{1}^{0}\right|} \log \left|G_{\chi}(0)+7\right|+\log \left|A_{2}(\chi)-A_{1}(\chi)\right|+\log \left|A_{1}(\chi)\right|\right. \\
+\log 2\} \frac{\left(s^{2}\left|z_{1}^{0}\right|^{2}-\left|z_{2}\right|^{2}\right) d \chi}{\left|s z_{1}^{0}\right|^{2}+\left|z_{2}\right|^{2}-2 s\left|z_{1}^{0} z_{2}\right| \cos \left(\chi-\arg z_{2}\right)} .
\end{gathered}
$$

Replacing $z_{1}^{0}$ by $z_{1}$, and $G_{x}$ by the right-hand side of (7) yields (5). 
Remark 2. $\boldsymbol{D}+\mathfrak{N}, \quad \mathfrak{N}=\left[\left|z_{k}\right|<\varepsilon\right], \quad \varepsilon>0$ sufficiently small, is a nonexistence domain. By the Hartogs theorem [20] every function regular in $\mathfrak{D}+\mathfrak{A}$ must be regular in $\left[\left|z_{1}\right|<r,\left|z_{2}\right|<s r\right]$.

The upper bound for $\left|f\left(r e^{i \varphi_{1}}, s_{1} r_{1} e^{i \varphi_{2}}\right)\right|, 0 \leqq \varphi_{k} \leqq 2 \pi, r_{1}<r, s_{1}<s$, which one obtains using (5) holds in $\left[\left|z_{1}\right|<r_{1},\left|z_{2}\right|<s_{1} r_{1}\right]$. On the other hand, one can construct functions $1 /\left(z_{1}-h\left(z_{2}\right)\right)$, e.g., $h\left(z_{2}\right)=0$ which are infinite at $(0,0)$ and regular in 2 .

Remark 3. For simplicity sake we consider here $\mathfrak{b}^{3}$ which is a sum of segments of analytic planes $\mathfrak{P}^{2}(\alpha)$. One can generalize our results replacing $\boldsymbol{\mathbf { D }}^{3}$ by $\tilde{\mathbf{D}}^{3}=\bigcup_{\alpha \subset \mathfrak{q}^{1}} \tilde{\mathfrak{F}}^{2}(\alpha)$, where $\tilde{\mathfrak{P}}^{2}(\alpha)$ are segments of analytic surfaces $z_{1}=p\left(\alpha, z_{2}\right)$, and $p\left(\alpha, z_{2}\right)$ satisfy certain conditions. In this case $\tilde{\mathfrak{B}}^{2}(\alpha)$ can have several intersection points.

We proceed now to the investigation of functions $f\left(z_{1}, z_{2}\right)$ in a domain

$$
\mathbf{B}=\bigcup_{\alpha \in \mathfrak{S}^{2}} \mathfrak{Q}^{2}(\alpha), \quad O \in \mathfrak{Q}^{2}(\alpha), \quad \mathfrak{Q}^{2}(\alpha) \subset \mathfrak{P}^{2}(\alpha),
$$

where $\mathfrak{S}^{2}$ is a simply connected region situated in the part

$$
0<\delta \leqq\left|\alpha+\frac{a_{10}}{a_{01}}\right| \leqq \delta^{-1}
$$

of the $\alpha$-plane, see $(6)$.

Definition. A function $f\left(z_{1}, z_{2}\right)$ which has the property that in every $\mathfrak{Q}^{2}(\alpha)$ of the domain $\mathbf{3}$, see $(15)$, it assumes every value not more than once will be called a function of the class $\boldsymbol{S}(\boldsymbol{\mathfrak { s }}), f \in \boldsymbol{S}(\boldsymbol{\mathfrak { S }})$.

If $f\left(z_{1}, z_{2}\right)$ has the above indicated property only in those $\mathfrak{D}^{2}(\alpha)$, which constitute the part $\boldsymbol{\nabla}^{3}=\bigcup_{\alpha \in \mathfrak{q}^{1}} \mathfrak{Q}^{2}(\alpha)$ of the boundary of $\mathfrak{\vartheta}$, we shall write: $f \in \boldsymbol{S}\left(\boldsymbol{\triangleright}^{3}\right)$.

We assume that $\mathfrak{D}^{2}(\alpha)$ is simply connected and that the boundary curve of its $z_{1}$-projection ${ }^{7}$ ) lies in the ring

$$
0<\varepsilon \leqq\left|z_{1}\right| \leqq \frac{1}{\varepsilon}
$$

Further let $z_{1}=w(\alpha ; \zeta), w(\alpha ; 0)=0$, be the function which maps the disc $|\zeta|<1$ onto the projection of $\Omega^{2}(\alpha)$ onto the $z_{1}$-plane.

Theorem 5.2. Let $f\left(z_{1}, z_{2}\right), f \in \boldsymbol{S}(\boldsymbol{3})$,

$$
f\left(z_{1}, z_{2}\right)=a_{10} z_{1}+a_{01} z_{2}+\ldots, \quad\left(z_{1}, z_{2}\right) \in \mathfrak{R}(O),
$$

be holomorphic in the (closed) domain $\overline{\mathbf{B}}^{8}$ ). Then the inequality

7) $z_{1}$-projection $=$ projection on the plane $z_{1}=$ const.

8) $\mathfrak{R}(O)$ the neighborhood of the origin. 


$$
\begin{gathered}
\left|w^{\prime}(\alpha ; 0)\left(a_{10}+\alpha a_{01}\right)\right| \frac{\left|\tilde{\zeta}\left(\alpha ; z_{1}\right)\right|}{\left(1+\left|\tilde{\zeta}\left(\alpha ; z_{1}\right)\right|\right)^{2}} \\
\leqq\left|f\left(z_{1}, z_{2}\right)\right| \leqq \mid w^{\prime}(\alpha ; 0)\left(a_{10}+\alpha a_{01}\right) \frac{\left|\tilde{\zeta}\left(\alpha ; z_{1}\right)\right|}{\left(1-\left|\tilde{\zeta}\left(\alpha ; z_{1}\right)\right|\right)^{2}}, \\
\alpha=\frac{z_{2}}{z_{1}}, \quad w^{\prime}(\alpha ; 0)=\left.\frac{d w(\alpha ; z)}{d z}\right|_{z=0}, \quad a_{10}+\alpha a_{01} \neq 0,
\end{gathered}
$$

where $\tilde{\zeta}\left(\alpha ; z_{1}\right)$ is the inverse of the function $w(\alpha ; \zeta)$ and $z_{1} \neq 0$.

Proof. In accordance with our assumptions every intersection $\mathfrak{D}^{2}(\alpha)$ can be mapped onto the unit circle. Let

$$
w(\alpha ; \zeta)=w^{\prime}(\alpha ; 0) \zeta+\ldots
$$

be the function element of the mapping function. The development of $f[w(\alpha ; \zeta), \alpha w(\alpha ; \zeta)]$ in $|\zeta| \leqq 1$ is

$$
f[w(\alpha ; \zeta), \alpha w(\alpha ; \zeta)]=\left(a_{10}+\alpha a_{01}\right) w^{\prime}(\alpha ; 0) \zeta+\ldots .
$$

According to our assumptions $a_{10}+\alpha a_{01} \neq 0$ and $0<\left|w^{\prime}(\alpha ; 0)\right|<\infty$. Since $f\left[z_{1}(w), \alpha z_{1}(w)\right]$ is a schlicht function, it holds

$$
\begin{gathered}
\left|w^{\prime}(\alpha ; 0)\left(a_{10}+\alpha a_{01}\right)\right| \frac{|\zeta|}{(1+|\zeta|)^{2}} \leqq|f(w(\alpha ; \zeta), \alpha w(\alpha ; \zeta))| \\
\leqq\left|w^{\prime}(\alpha ; 0)\left(a_{10}+\alpha a_{01}\right)\right| \frac{|\zeta|}{(1-\mid \zeta)^{2}} .
\end{gathered}
$$

See [15] p. 88. Setting $\alpha=z_{2} / z_{1}, \quad \zeta=\tilde{\zeta}\left(z_{2} / z_{1}, z_{1}\right)$, we obtain the inequality (19).

Theorem 5.3. Let $f\left(z_{1}, z_{2}\right), f(0,0)=0$, be regular in the domain 2 , see (2). If $f \in \boldsymbol{S}\left(\mathbf{D}^{3}\right)$ and $f$ has the development (18) at the origin, then the inequality

$$
\log \left|f\left(z_{1}, z_{2}\right)\right| \leqq
$$

$$
\begin{array}{r}
\frac{1}{2 \pi} \int_{\chi=0}^{2 \pi}+\log \frac{r^{2}\left|a_{10}+s e^{i \chi} a_{01}\right|\left|z_{1}\right|}{\left(r-\left|z_{1}\right|\right)^{2}} \frac{\left(s^{2}\left|z_{1}\right|^{2}-\left|z_{2}\right|^{2}\right) d \chi}{s^{2}\left|z_{1}\right|^{2}+\left|z_{2}\right|^{2}-2 s\left|z_{1}\right|\left|z_{2}\right| \cos \left(\chi-\arg z_{2}\right)} \\
s=|\alpha| .
\end{array}
$$

holds.

Proof. The function $f\left(z_{1}, s e^{i \chi} z_{1}\right)$ is schlicht in the disc $\left|z_{1}\right| \leqq r$ and has the development

$$
f\left(z_{1}, s e^{i \chi} z_{1}\right)=z_{1}\left(a_{10}+a_{01} s e^{i \chi}\right)+z_{1}^{2}(\ldots)+\ldots
$$

at $z_{1}=0$. 
According to the known results about schlict functions of one variable, it holds

$$
\left|f\left(z_{1}, s e^{i \chi} z_{1}\right)\right| \leqq \frac{\left|\left(a_{10}+a_{01} s e^{i \chi}\right) r^{2} z_{1}\right|}{\left(r-\left|z_{1}\right|\right)^{2}},
$$

see [15] p. 89. Let $\left(z_{1}^{0}, z_{2}^{0}\right)$ be a point in the disc $\left|z_{2}\right| \leqq s\left|z_{1}^{0}\right|$, intersection of 2 and $z_{1}=z_{1}^{0}$. At every point $z_{2}=s\left|z_{1}^{0}\right| e^{i \chi}$ of the boundary, the inequality (25) holds. Therefore, by Poisson-Jensen formula and replacing $z_{1}^{0}, z_{2}^{0}$ by $z_{1}, z_{2}$, we obtain the inequality (23).

\section{Some properties of the function $f\left(z_{1}, z_{2}\right)$ (given by its function element) in a bounded domain}

The generalization to the case of two variables of relations between the coefficients $a_{m}$ of the series development of

$$
f(z)=\sum_{n=0}^{\infty} a_{n} z^{n}
$$

and the location of the poles of $f$ (Hadamard's theory) is discussed in $\left.[13]^{9}\right)$. In the following we continue these considerations.

We assume that the origin in every plane (5.1) coincides with $z_{1}=z_{2}=0$. Let $f\left(z_{1}, z_{2}\right)$ be a meromorphic function regular at the origin. In the plane $\mathfrak{P}^{2}(\alpha)$, see (5.1), the function

$$
f=\sum_{m, n=0}^{\infty} a_{m n} z_{1}^{m} z_{2}^{n}
$$

becomes $F_{\alpha}\left(z_{1}\right) \equiv f\left(z_{1}, \alpha z_{1}\right)$ a function of one complex variable

$$
F_{\alpha}\left(z_{1}\right)=\sum_{N=0}^{\infty} z_{1}^{N}\left(\sum_{\varkappa=0}^{N} a_{N-\varkappa, \varkappa} \alpha^{\varkappa}\right) \equiv \sum_{N=0}^{\infty} z_{1}^{N} a_{N}(\alpha)=
$$

Following Hadamard's procedure in one variable, we introduce the functions

$$
l_{\mu}(\alpha)=\lim _{\beta \rightarrow \alpha} \lim _{N \rightarrow \infty}\left[D_{N}^{(\mu)}(\beta)\right]^{1 / N},
$$

$\left.{ }^{9}\right)$ The results in this direction can be generalized to the case of harmonic functions and solutions of differential equations and systems of equations in three and more variables (see [13]). 


$$
D_{N}^{(\mu)}(\beta)=\left|\begin{array}{cccc}
a_{N}(\beta) & a_{N+1}(\beta) & \ldots & a_{N+\mu}(\beta) \\
a_{N+1}(\beta) & a_{N+2}(\beta) & \ldots & a_{N+\mu+1}(\beta) \\
\cdot & \cdot & \ldots & \cdot \\
\cdot & \cdot & \ldots & \cdot \\
\cdot & \cdot & \ldots & \cdot \\
a_{N+\mu}(\beta) & a_{N+\mu+1}(\beta) & \ldots & a_{N+2 \mu}(\beta)
\end{array}\right|
$$

With a meromorphic function $F_{\alpha}\left(z_{1}\right)$ of one complex variable $z_{1}$, one associates pairs of numbers and integers $\left(\varrho_{n}, k_{n}\right), n=1,2, \ldots, k_{n}$, where $\varrho_{n}$ are radii of those circles on which the poles of $F_{a}$ are located, and $k_{n}$ is the number of poles (counted with appropriate multiplicity) which lies on the circle $\left|z_{1}\right|=\varrho_{n} \quad$ (Hadamard [19] p. 120).

In order to apply Hadamard's approach, we introduce the sets $\mathfrak{I}_{n}^{2}$ to be described below.

For every $\alpha$ the intersection of singularity surfaces of $f$ with $\mathfrak{P}^{2}(\alpha)$ forms at most a denumerable set of points which lie on the circle

(4) $\quad r \equiv\left(\left|z_{1}\right|^{2}+\left|z_{2}\right|^{2}\right)^{1 / 2}=\varrho_{n}(\alpha), \quad \varrho_{n}(\alpha)<\varrho_{n+1}(\alpha), \quad\left(z_{1}, z_{2}\right) \in \mathfrak{P}^{2}(\alpha)$.

To every $\varrho_{n}(\alpha)$ corresponds a finite number $k_{n}(\alpha)$, the number of poles (counted with corresponding multiplicity) lying on the circle $r=\varrho_{n}(\alpha)$ in the plane $\mathfrak{P}^{2}(\alpha)$.

Let $\alpha$ vary. We denote by $\mathfrak{I}_{n}^{2}$ the set of singular points of $f\left(z_{1}, z_{2}\right)$ which lie on the boundary of the circular domain $\left(\left|z_{1}\right|^{2}+\left|z_{2}\right|^{2}\right)^{1 / 2}$ $=\varrho_{n}\left(z_{2} / z_{1}\right)$. (In general, $\mathfrak{I}_{n}^{2}$ consists of a number of disconnected sets.)

Using the previously mentioned results, we obtain information about the three-dimensional manifolds on which the $\mathfrak{I}_{n}^{2}$ are located.

An example of the application of Hadamard's results to the case of two variables will be discussed in the following. (Also comp. [13] § 3.)

Let $\mathfrak{Q}^{2}$ be a simply connected domain in the $\alpha$-plane and let (5.6) be the development of the function $f$, see (5.4), in the plane $z_{2}=\alpha z_{1}, \alpha=s e^{i \chi}$. We determine the corresponding $l_{\mu}(\alpha)$, see (3), and let

$$
\varrho_{n}(\alpha) \equiv l_{n}(\alpha) / l_{n-1}(\alpha) \text {. }
$$

Theorem 6.1. Suppose that the $\varrho_{n}(\alpha), \alpha \in \mathfrak{\Omega}^{2}$, can be subdivided into denumerably many subsequences

$$
\left[\varrho_{p}(\alpha), \varrho_{p^{+1}}(\alpha), \ldots, \varrho_{p_{p^{+1}}-1}(\alpha)\right], \quad p=1,2, \ldots,
$$

such that

$$
\hat{\varrho}_{p}(\alpha) \doteq \varrho_{n_{p}}(\alpha)=\varrho_{n_{p}+1}(\alpha)=\ldots=\varrho_{n_{p+1}-1}(\alpha)<\varrho_{n_{p+1}}(\alpha) .
$$


If $n_{p+1}(\alpha)-n_{p}(\alpha)=k_{p}=$ const for $\alpha \in \mathfrak{D}^{2}$, then in the three-dimensional segment of circular domains

$$
\left|z_{1}\right|^{2}+\left|z_{2}\right|^{2}=\hat{\varrho}_{p}\left(\frac{z_{2}}{z_{1}}\right), \quad \frac{z_{2}}{z_{1}} \in \mathfrak{D}^{2},
$$

lie $k_{p}$ (two-dimensional) segments of singular sets $\mathfrak{I}_{n}^{2}$. Here $k_{p}=$ $\varrho_{n_{p+1}}(\alpha)-\varrho_{p}(\alpha)$. These $\mathfrak{I}_{n}^{2}$ are the only singularity surfaces for $z_{2} / z_{1} \in \mathfrak{Q}^{2}$.

Obviously, proceeding in a similar manner, many theorems of Hadamard's theory in one variable lead to the theorems about functions of two complex variables.

The theory of orthogonal functions is an effective tool for the study of the properties of an analytic function of one complex variable given by its series development. In the following we shall describe two different criteria for the function element

$$
f(z)=\sum_{n=0}^{\infty} b_{n} z^{n}
$$

to have an analytic continuation into a given simply connected domain $\mathfrak{B}^{2}$ which inc'udes the origin.

We begin with a description of two sets of orthogonal functions.

1. Let $\mathfrak{G 5}^{2}, \mathfrak{B}^{2}, \mathfrak{F}^{2} \subset \mathfrak{B}^{2}$, be two simply connected domains in the $z$-plane. There exists a set of orthogonal functions, complete in $\left(S^{2}\right.$, such that

$$
\iint_{\mathbb{B}^{2}} \varphi_{\nu}(z) \overline{\varphi_{\mu}(z)} d \omega=k_{\nu} \delta_{\nu_{\mu}}, \quad \iint_{\sigma^{2}{ }^{2}} \varphi_{\nu}(z) \overline{\varphi_{\mu}(z)} d \omega=\delta_{\nu_{\mu}} .
$$

Here $d \omega=d x d y, \quad \delta_{v_{\mu}}=0 \quad$ for $\quad v \neq \mu \quad$ and $\quad \delta_{v v}=1 . \quad$ [9] p. 41, [10] p. 14.

2. Let the origin $O$ be the interior point of $\mathfrak{B}^{2}$. There exists a complete set of functions $\psi_{n}(z)$, orthonormal in $\mathfrak{B}^{2}$ and possessing the property that

$$
\psi_{n}(z)=\sum_{v=n-1}^{\infty} a_{n v} z^{v}
$$

[9] p. 28.

Using the set $\left\{\varphi_{\nu}(z)\right\}$, one proves the following:

Theorem 6.2. Let $\mathfrak{B}^{2}$ be a bounded simply connected domain containing the circle $|z| \leqq r$. There exists a sequence of Hermitian forms

$$
H_{n}\left(x_{\nu}, \bar{x}_{\mu}\right)=\sum_{\nu=0}^{\infty} \sum_{\mu=0}^{\infty} A_{\nu \mu}^{(n)} x_{\nu} \bar{x}_{\mu}
$$


with the following property: Let $f(z)$, given by (9), be regular in $[|z| \leqq r]$. If $f(z)$ is regular in $\mathfrak{B}^{2}$ and

$$
J_{\mathfrak{B}^{2}}(f) \equiv \int_{\mathfrak{B}^{2}} \int_{\mid} \mid f^{2} d \omega<\infty,
$$

then

$$
\lim _{n \rightarrow \infty} H_{n}\left(\frac{b_{v}}{d_{v}}, \frac{\overline{b_{\mu}}}{d_{\mu}}\right), \quad d_{v}=\frac{1}{r^{\nu+1}}\left(\frac{\nu+1}{\pi}\right)^{1 / 2},
$$

exists. Conversely, if (13) exists, $f(z)$ may be continued analytically throughout $\mathfrak{B}^{2}$ and the inequality

$$
|f(z)| \leqq \frac{1}{\pi^{1 / 2}} \frac{1}{r(z)}\left(\lim _{n \rightarrow \infty} H_{n}\left(\frac{b_{v}}{d_{v}}, \frac{\overline{b_{v}}}{d_{\mu}}\right)\right)^{1 / 2}, \quad z \in \mathfrak{B}^{2},
$$

holds, where $r(z)$ is the distance of $z$ to the boundary of $\mathfrak{B}^{2} . \quad[10]$ p. 18.

From the above follows:

Theorem 6.3. Let

$$
f\left(z_{1}, z_{2}\right)=a_{00}+a_{10} z_{1}+a_{01} z_{2}+\ldots
$$

be a function regular in the (closed) hypersphere

$$
\mathbb{0}=\left[\left|z_{1}\right|^{2}+\left|z_{2}\right|^{2} \leqq r^{2}\right] .
$$

Further, let $\mathbf{B}$ be a domain containing $\overline{\boldsymbol{G}}$. We assume that

$$
\boldsymbol{B} \cap \mathfrak{P}^{2}(\alpha)=\mathfrak{S}^{2}(\alpha)
$$

is simply connected and that $\mathfrak{S}^{2}(\alpha)$ depends continuously on $\alpha$. There exists a sequence of Hermitian form

$$
H_{n}\left(\alpha ; x_{\nu}, x_{\mu}\right)=\sum_{\nu=0}^{\infty} \sum_{\mu=0}^{\infty} A_{\nu \mu}^{(n)}(\alpha) x_{\nu} \bar{x}_{\mu}
$$

with the following property: If $f\left(z_{1}, z_{2}\right)$ is regular in $\mathfrak{S}^{2}(\alpha)$ and $J_{\mathbb{E}^{2}(\alpha)}\left(F_{\alpha}(z)\right)<\infty$, see $(5.6)$, then

$$
\boldsymbol{H}(\alpha) \equiv \lim _{n \rightarrow \infty} H_{n}\left(\alpha ; \frac{\sum_{x=0}^{v} a_{\nu-,, r} \alpha^{*}}{d_{\nu}}, \overline{\left(\frac{\sum_{\nu=0}^{\mu} a_{\mu-\varkappa, \%} \alpha^{*}}{d_{\mu}}\right)}\right)<\infty .
$$

Conversely, if (18) holds for $\alpha$ belonging to the neighborhood $\left|\alpha-\alpha_{0}\right|<\varepsilon$, $\varepsilon>0$, then $f\left(z_{1}, z_{2}\right)$ can be continued to $\mathfrak{S}^{2}\left(\alpha_{0}\right)$ and the function $f\left(z_{1}, \alpha_{0} z_{1}\right)$ is square integrable in $\mathbb{S}^{2}\left(\alpha_{0}\right)$. 
Let $\mathfrak{\sim}^{2}$ be the domain in the $\alpha$-plane introduced on p. 15 and let $\mathfrak{q}^{1}$ be its boundary curve. We assume that in every $\mathfrak{P}^{2}(\alpha), \alpha \in \mathfrak{D}^{2}$, lies a domain $\mathfrak{D}^{2}(\alpha), \quad O \in \mathfrak{D}^{2}(\alpha)$. The boundaries $\mathfrak{D}^{1}(\alpha)$ of $\mathfrak{D}^{2}(\alpha)$ are supposed to be differentiable curves which vary continuously in $\alpha$. Finally let

$$
\mathfrak{S S}^{2}=\bigcup_{\alpha \in \mathfrak{q}^{2}} \mathfrak{D}^{1}(\alpha), \quad \mathfrak{O}=\bigcup_{\alpha \in \mathfrak{\Sigma}^{2}} \mathfrak{D}^{2}(\alpha)
$$

$\mathfrak{g}_{1}^{3}+\mathfrak{g}_{2}^{3}, \quad$ where

$$
\mathbf{g}_{1}^{3}=\bigcup_{\alpha \in \mathfrak{q}^{1}} \mathfrak{D}^{2}(\alpha), \quad \mathfrak{g}_{2}^{3}=\bigcup_{\alpha \in \mathbb{Q}^{2}} \mathfrak{D}^{1}(\alpha),
$$

is the boundary of 2 and let

$$
\mathfrak{S S}^{2}=\mathfrak{g}_{1}^{3} \cap \mathfrak{g}_{2}^{3} .
$$

Definition. Suppose that through every point $\left(z_{1}^{(p)}, z_{2}^{(p)}\right)$ of a subdomain $\mathfrak{P}$ of $\mathfrak{P}$ passes an analytic surface

$$
\mathfrak{I}^{2}=\left[z_{k}=g_{k}\left(z_{1}^{(p)}, z_{2}^{(p)} ; \zeta\right)=g_{\dot{k}}(\zeta)\right], \quad k=1,2, \quad|\zeta| \leqq s:
$$

whose boundary curve

$$
\mathfrak{t}^{1}=\left[z_{k}=g_{k}\left(s e^{i \psi}\right), 0 \leqq \psi \leqq 2 \pi\right]
$$

lies in $\mathfrak{g}_{1}^{3}-\mathfrak{S}^{2}$. Then we shall call $\mathfrak{F}$ subordinated to $\mathfrak{S}^{2}$. We assume that $\mathfrak{I}^{2}$ intersects every lamina $\mathfrak{D}^{2}(\alpha)$ in one and only one point, and that

$$
0<\frac{d \alpha(\psi)}{d \psi}<\infty .
$$

Theorem 6.4. Let the point $(z)=\left(z_{1}^{(p)}, z_{2}^{(p)}\right)$ of $\mathfrak{R}, \quad$ (a subdomain of 2 subordinated to $\left.\mathbb{S}^{2}\right)$ lie in the analytic surface $\mathfrak{I}^{2}$, see (21).

$$
\mathrm{t}_{1}=\mathfrak{I}^{2} \cap \mathfrak{g}_{1}^{3}=\left[z_{k}=h_{k}\left(s e^{i \chi}\right), s \text { fixed }, 0 \leqq \chi \leqq 2 \pi\right]
$$

is the boundary of $\mathfrak{I}^{2}$. Let $f\left(z_{1}, z_{2}\right)$ be an analytic function regular in 2. Then

$$
\begin{gathered}
\log |f(z)| \equiv \log |f(g(\zeta))| \\
\leqq \frac{1}{2 \pi^{3 / 2}} \int_{0}^{2 \pi} \frac{\log (H(\alpha(\psi)))^{1 / 2}}{\varrho(\alpha(\psi))} \frac{s^{2}-|\xi|^{2}}{s^{2}+|\xi|^{2}-2 s|\xi| \cos (\psi-\chi)} d \psi \\
-\sum_{\nu=1}^{d_{\mu}} \log \left|\frac{s^{2}-\bar{\xi}_{\nu} \xi}{s\left(\xi-\xi_{v}\right)}\right|
\end{gathered}
$$


Here $\varrho(\alpha)$ is the distance of $\mathfrak{T}^{2} \cap \mathfrak{P}^{2}(\alpha)$ from $\mathfrak{S}^{2}, \quad H(\alpha)$ is the expression (18), where $H_{n}$ are the Hermitian forms, see (17), which were associated with $\mathscr{D}^{2}(\alpha), \alpha \in \mathfrak{q}^{1} ; s e^{i \psi}, 0 \leqq \psi \leqq 2 \pi$, is the image of $\mathrm{t}^{1}$ in the $\zeta$ plane, and $\zeta_{v}, v=1,2, \ldots$, are the images of the zero points of $f$ in $\mathfrak{I}^{2}$.

Proof. According to our assumptions at every point of $\mathfrak{F}^{2}(\alpha) \cap \mathfrak{I}^{2}$ $=\left(z_{1}, \alpha z_{1}\right)$

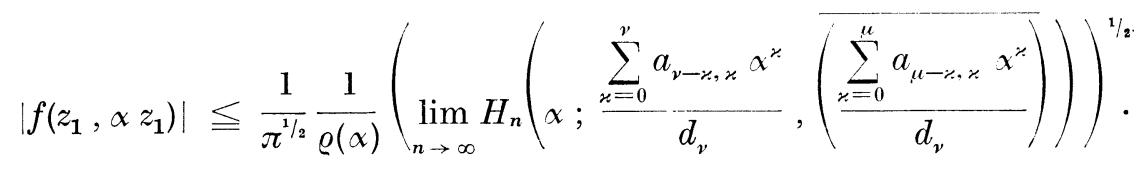

To every point $\left(h\left(s e^{i x}\right)\right)$ corresponds a point $(z(\alpha)) \in \mathrm{t}^{1}$ and vice versa and the function $\chi=\chi(\alpha)$ is differentiable. We consider now the function $f(g(\xi))$. At every boundary point

$$
f\left(z_{1}, \alpha z_{1}\right)=f\left(g\left(s e^{i \chi}\right)\right)
$$

satisfies the inequality (25). By the Poisson-Jensen formula (24) follows.

Using the results of [14] p. 240, Schiffer and Siciak [23] gave a constructive criterium which yields the necessary and sufficient conditions in order that a function $p(\zeta)$, given by its function element, can be analytically continued into a larger prescribed domain.

Using these results, one obtains instead of (18) different necessary and sufficient conditions in order that a function given by (15) is square integrable in $\mathfrak{Q}^{2}(\alpha)$ (see Theorem 6.2).

Remark 1. In $\S 5$ we consider the case of functions which are schlicht or omit two values in every lamina. Instead of these functions, one obviously can use other families of functions, e.g., $p$-valent functions. functions which map the unit circle on star or convex domains, etc.

Stanford University

California, U.S.A. 


\section{Bibliography}

[1] Ahlfors, L. V.: An extension of Schwarz's lemma. - Trans. Amer. Math. Soc. 43,1938 , pp. $359-364$.

[2] Bergman, S.: Über ausgezeichnete Randflächen in der Theorie der Funktionen von zwei komplexen Veränderlichen. - Math. Ann. 104, 1931, pp. 611-636.

[3] - - Über eine in gewissen Bereichen mit Maximumfläche gültige Integraldarstellung der Funktionen zweier komplexer Variabler. I. - Math. Z. 39, 1935, pp. $76-94$.

[4] -》- Über eine in gewissen Bereichen mit Maximumfläche gültige Integraldarstellung der Funktionen zweier komplexer Variabler. II. - Math. Z. 39,1935 , pp. $605-608$.

[5] -"- Über eine Integraldarstellung der Funktionen zweier komplexer Veränderlichen. - Recueil Mathématique [Математический Сборник] (Nouvelle Série) 1 (43), 1936, pp. 851-862.

[6] -》- Über eine Abschätzung von meromorphen Funktionen zweier Veränderlicher in Bereichen mit ausgezeichneter Randfläche. - Académie des Sciences de l'URSS. Filiale Géorgienne. Travaux de l'Institut Mathématique de Tbilissi 1, 1937, pp. 187-204.

[7] -»- Über das Verhalten der Funktionen von zwei komplexen Veränderlichen in Gebieten mit einer ausgezeichneten Randfläche. - Mathematica. Publicată de Seminarul de Matematici al Universităţei Cluj 14, 1938, pp. $107-123$.

[8] -»- Über meromorphe Funktionen von zwei komplexen Veränderlichen. . Compositio Math. 6, 1939, pp. 305-335.

[9] -"- Sur les fonctions orthogonales de plusieurs variables complexes avec les applications à la théorie des fonctions analytiques. - Interscience Publishers, New York, 1941. = Mémorial des Sciences Mathématiques CVI, Gauthier-Villars, Paris, 1947.

[10] - - The kernel function and conformal mapping. - Mathematical Surveys V, American Mathematical Society, New York, 1950.

[11] -»- Bounds for analytic functions in domains with a distinguished boundary surface. - Math. Z. 63, 1955, pp. 173-194.

[12] - - On value distribution of meromorphic functions of two complex variables. - Studies in mathematical analysis and related topics: Essays in honor of George Pólya, Stanford Studies in Mathematics and Statistics IV, Stanford Univeristy Press, Stanford (Calif.), 1962, pp. 25-37.

[13] - - On the coefficient problem in the theory of a system of linear partial differential equations. - J. Analyse Math. 11, 1963, pp. 249-274.

[14] Bergman, S., and M. Schiffer: Kernel functions and conformal mapping. Compositio Math. 8, 1951, pp. 205-249. 
[15] Bieberbach, L.: Lehrbuch der Funktionentheorie. Band II: Moderne Funktionentheorie. - B. G. Teubner, Leipzig/Berlin, 1927.

[16] Charzý́ski, Z.: Bounds for analytic functions of two complex variables. - Math. Z. 75,1961 , pp. 29-35.

[17] Dienes, P.: The Taylor series. An introduction to the theory of functions of a complex variable. - Clarendon Press, Oxford, 1931. = Dover Publications, New York, 1957.

[18] Dinghas, A.: Vorlesungen über Funktionentheorie. - Grundlehren der mathematischen Wissenschaften 110, Springer-Verlag, Berlin/Göttingen/Heidelberg, 1961.

[19] Hadamard, J.: Essai sur l'étude des fonctions données par leur développement de Taylor. - J. Math. Pures Appl. (4) 8, 1892, pp. 101-186.

[20] Hartogs, F.: Zur Theorie der analytischen Funktionen mehrerer unabhängiger Veränderlichen, insbesondere über die Darstellung derselben durch Reihen, welche nach Potenzen einer Veränderlichen fortschreiten. - Math. Ann. 62,1906, pp. $1-88$.

[21] Nevanlinna, R.: Le théorème de Picard-Borel et la théorie des fonctions méromorphes. - Collection de Monographies sur la Théorie des Fonctions, Gauthier-Villars et Cie, Paris, 1929.

[22] - - Eindeutige analytische Funktionen. - Grundlehren der mathematischen Wissenschaften XLVI, Verlag von Julius Springer, Berlin, 1936.

[23] Schiffer, M., and J. Siciak: Transfinite diameter and analytic continuation of functions of two complex variables. - Studies in mathematical analysis and related topies: Essays in honor of George Pólya, Stanford Studies in Mathematics and Statistics IV, Stanford University Press, Stanford (Calif.), 1962, pp. 341-358.

[24] ŚLadkowska, J.: Bounds of analytic functions of two complex variables in domains with the Bergman-Shilov boundary. - Pacific J. Math. 12, 1962, pp. 1435-1451.

[25] - - Bounds of analytic functions of two complex variables in domains with the Bergman-Shilov boundary. - Proc. Nat. Acad. Sci. U.S.A. 49, 1963, pp. $217-219$. 